\title{
AUTOMATIC MULTI-PARAMETRIC DIMENSIONAL AND GEOMETRIC INSPECTION OF ROTARY SYMMETRICAL PARTS LIKE BEARING RINGS
}

\author{
Cioboată Daniela ${ }^{1}$, Soare Adrian'2, Stanciu Dănuțin ${ }^{1}$ Abălaru Aurel ${ }^{1}$, Logofătu Cristian ${ }^{1}$ \\ ${ }^{1}$ National Institute of Research and Development in Mechatronics and Measurement Technique, Pantelimon Road \\ no. 6-8, 021631 Bucharest, Romania, \\ 2 SC COMIS SRL, Road Nicolae Iorga no. 83, Câmpina, 106400, Prahova, Romania \\ cioboatadoina@yahoo.com,adyse_prod@yahoo.com,danutstanciu@yahoo.com,aurel.abalaru@gmail.com, \\ cristilogofatu@yahoo.com
}

\begin{abstract}
The article presents the concept of a modular, multi-parametric measurement equipment, with large measurement range, automatic measurement cycle, for quality inspection of symmetrical parts like bearing rings. The article presents also a calibration method that allows to use a small number of standards for a large measuring range $(150 \mathrm{~mm})$ and a wide range of dimensional-type parts. The experiments made on the functional model proves the capability and functionality of the system. The obtained results will be presented and discussed. The aim of the described works was to develop an inspection system that will significantly increase the economic efficiency of bearing rings production and reduces the number of calibration standards required for calibration.
\end{abstract}

Keywords: Bearing Rings, Dimensional Inspection, Calibration, Modular Structure, Mechatronics.

\section{Introduction}

Today, requirements for form and positional tolerance measurements of work pieces in the metalworking industry are growing. For example, in bearing industry, bearing rings and rolling elements are required to operate at tougher conditions (operating speeds and temperatures) and consequently the dimensional precision and surfaces quality are very important to improve the dynamical performances. It is very important for bearings quality to improve the uniformity and the repeatability in the manufacturing process. A wellorganized quality control system based on smart, flexible and precision measuring machines is an important key element in the production of reliable and high-performance products. Furthermore, automation of measurement systems provides an important source of information on production processes and decrease considerable the measurement time.

Among the most important characteristics that determine the quality of the rolling bearings are: dimensional and geometrical accuracy of the bearing rings raceway, the outer diameter of the outer ring, the inner diameter of inner ring, the rings width, the rotation accuracy [3].

The manufacturing process of bearings usually consists of consecutive operations when the shape of the ring is obtained through primary machining followed by hardening and grinding or honing.

Dimensions, shapes and surfaces quality of turned rings are important to the quality of finished products. In the absence of rigorous inspections during the turning process, the quality of the bearing components can be compromised. Predictable repeatability in the manufacturing process is crucial in ensuring the performances of bearings.

The diversity of bearings forms and dimensions requires choosing some adequate criteria for measurement instrumentation design. Modular construction and flexibility of measurement instrumentation are two of the important design criteria. With the evolution of CNC manufacturing machines increased requirements for quality control in terms of accuracy, frequency, measurement cycle duration and statistical data processing. Development of smart, flexible and high level of automation equipment for manufactured parts quality inspection is one of the main requirements for the technological improvements of the bearings manufacturing systems [1].

Based on analysis of manufacturing technology of bearing rings, methods and instruments for their dimensional measurement, this paper recommends a multiparametric measurement equipment, with large measurement range, which uses the measurement method based on a rotary table and needs a small number of calibration standards. 


\section{Overview of the Bearing Measurement Instruments}

Today, due the wide variety of shapes and sizes, the measurement of dimensional and geometric deviation of rotary simetrical parts like bearing rings is generally made with universal equipment and devices and less with specialized equipment. For measuring rotary symmetrical parts, most often are used systems based on classic circular profile measurement or coordinate measurement schemes. Coordinate measuring machines, roundness measuring machines, contour measurement machines or length measuring machines are commonly used to inspect the quality of bearing rings, especially in metrological laboratories (fig. 1).

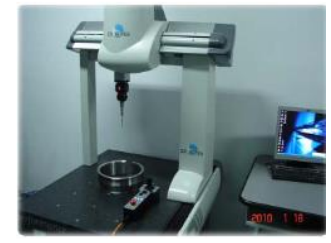

a. Coordinate measuring machine - DEA

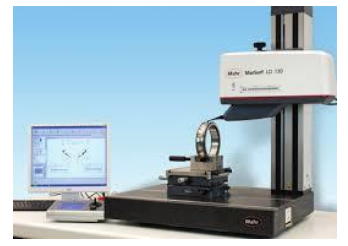

c. Contour measurement MarSurf LD 130

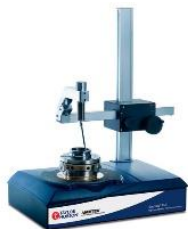

b. Surtronic R series Taylor Hobson

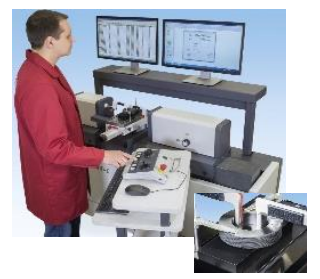

d. Universal lengths measuring machine Mahr
Figure 1. Universal measuring equipment

Many manufacturers use for measurement on production flow bench devices with dial indicators which need to be calibrated periodically. This control technology requires a great number of standards, is time consumption and does not allow the statistical control. Figure 2 shows some measuring devices currently used by bearings manufacturers.
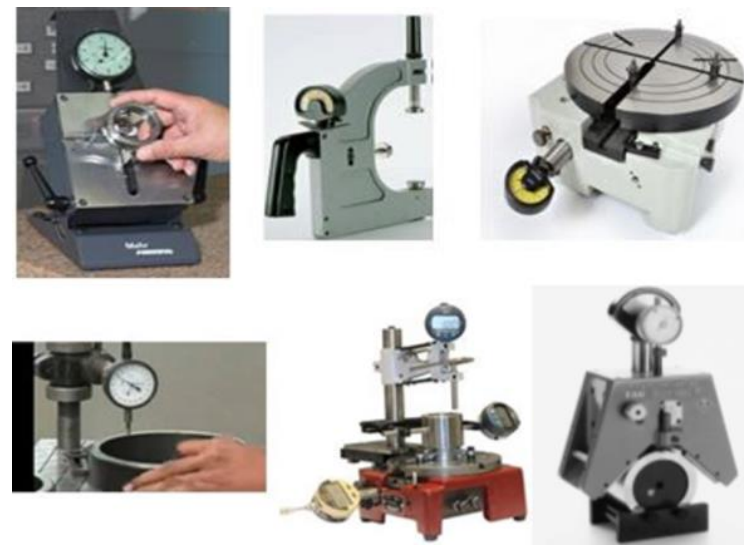

Figure 2. Bench measurement devices
Due to the rapid technological development, as well as stronger competition on global market, the trend is to replace these inspection technologies with innovative, intelligent control techniques to achieve greater productivity, higher quality of bearings and lower manufacturing costs.

Different specialized measurement equipment has been developed in automatic mode. Most, however, have a small measuring range, allow for a small number of parameters, generally in a single section, and require a large number of standards for calibration.
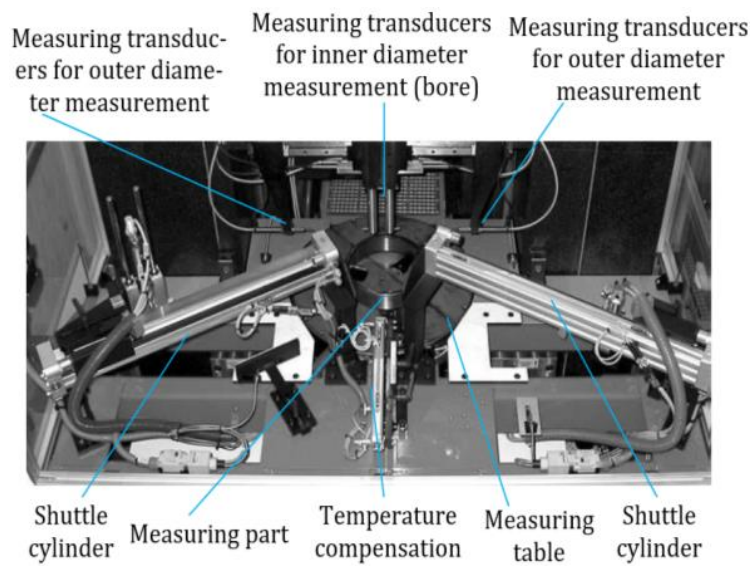

a. Measuring device for bearing inner and outer rings - MMQ 160B - SKF

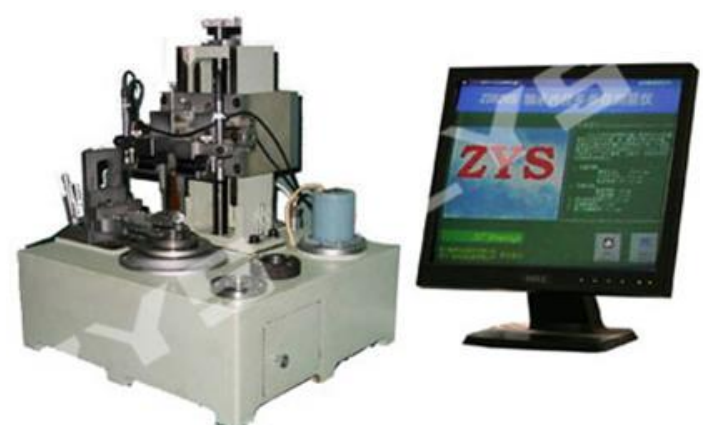

b. Equipment for bearing inner and outer diameter measurement - ZYS

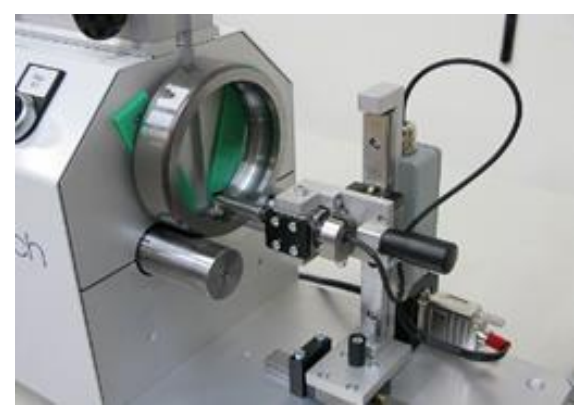

c. Inner diameter measurement - Bearing Scan 100 American stress technologies

Figure 3. Bearing rings measurement specialized measurement equipment

As shown in the above examples for the measurement of geometric values, contact methods 
are widely used. The advantages of these methods are the very high accuracy and reproducibility of the measurement. However, there is interest in development of contactless optoelectronic measuring techniques mainly because of the very high measurement speeds potentially. Their main drawback is that the instruments based on them can generate faulty measurements due to the presence of dirt, metal particles, machining fluids and also the part reflexivity [1] [4].

\section{Presentation of Own Solution}

In this paper we present a new architecture of an intelligent, flexible, multi-functional equipment which can be used for rolling bearing rings measurement.

The range of inspected parts included rotary symmetrical parts, like rings for cylindrical roller bearings rings, with inner diameters of $45 \ldots 180 \mathrm{~mm}$, outer diameters of $50 \ldots 200 \mathrm{~mm}$ and width up to 50 $\mathrm{mm}$.

Technical drawings showing the inspecting details for one type of inner bearing ring and one type of outer bearing ring is shown in Figure 4.

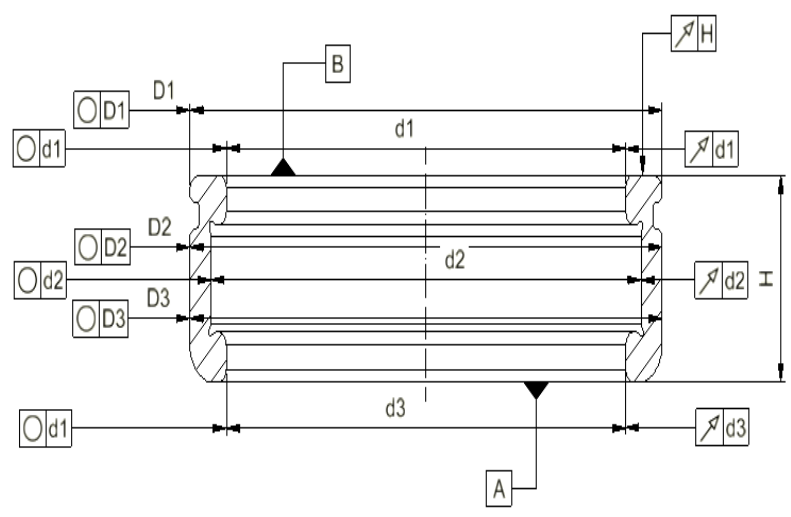

a. Inspecting parameters for outer bearing ring

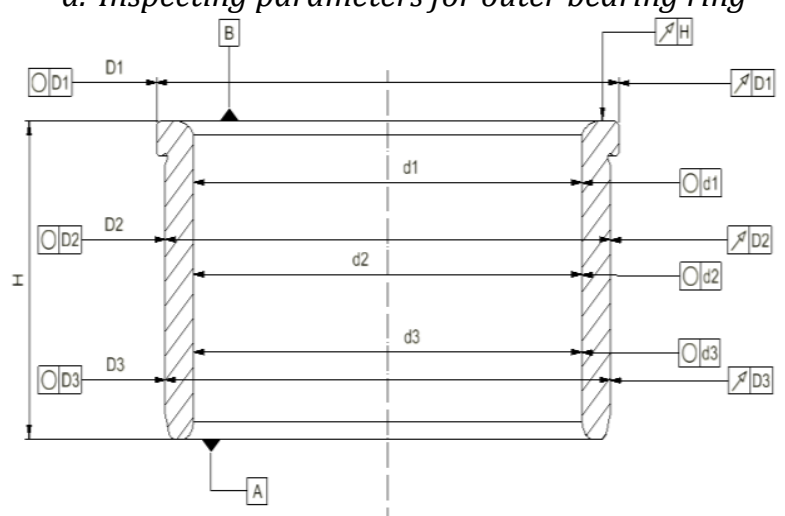

b. Inspecting parameters for inner bearing ring Figure 4. Measured parameters
The measurement tasks are (see table 1):

- Measurement of the inner and outer diameters (deviations and absolute values) (simultaneously in maximum 3 sections)

- Measurement of roundness of inner and outer diameters (simultaneously in maximum 3 sections);

- Measurement of run-out of inner or outer diameters (simultaneously in maximum 3 sections)

- Measurement of the bearing rings thickness deviation (axial circumferențial runout).

The main technical problem to be solved during development of this equipment was to make it flexible enough to allow measurement of bearing rings with various sizes and shapes, in multiple sections simultaneous, without the need for a large number of calibration standards. The equipment has a modular hardware and software structure for increasing functional flexibility to be able to adapt rapidly to changes in production [6].

Table 1. Measurement tasks

\begin{tabular}{|c|c|c|}
\hline $\begin{array}{c}\text { Inner and outer } \\
\text { diameter } \\
\text { measurement in } \\
\text { multiple planes } \\
\text { measurement in multiple } \\
\text { planes }\end{array}$ & $\begin{array}{c}\text { Inner and outer roundness } \\
\text { means }\end{array}$ \\
\hline & \\
\hline
\end{tabular}


Detail A: Inner and axial measurement modules
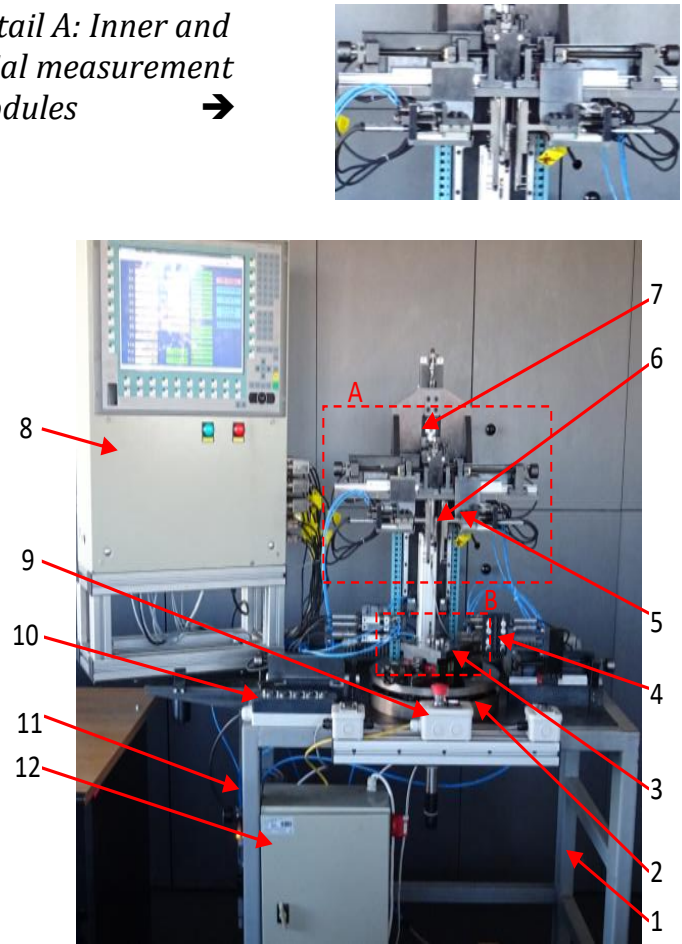

Detail B: Centering system

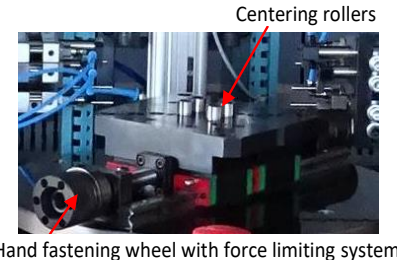

Figure 5. Equipment for bearing ring measurement

The developed measurement equipment consists of the following systems as shown in figure 5: base table (1); rotary table (2); adjustable centering system (3); outer diameters measurement modules (4); inner diameters measurement modules (5); axial measurement module (6); vertical column (7); data acquisition, processing and display unit (8); console with emergency stop button (9); console with buttons for manual controls (10); pneumatic system (11); electrical box; integrated software for the entire calibration and measurement process.

The arrangement of the probes and measurement principle is presented in figure 6. Centering and fastening of the bearing ring on the rotary table is made manually, with a four points flexible device which allows fixing the rings both on the inner or outer diameter (see Figure 5- Detail B and Figure 6). Fixing must ensure controlled gripping forces to prevent rings deformation [3]. Gripping force control is achieved by elastic force limiting coupling element integrated in hand fastening wheel (figure 5- Detail B).

For constructive reasons, the radial measurements are made with intermediate probing elements (Figure 7), adjustable in both radial and axial directions, to allow the measurement of a wide range of bearing rings.

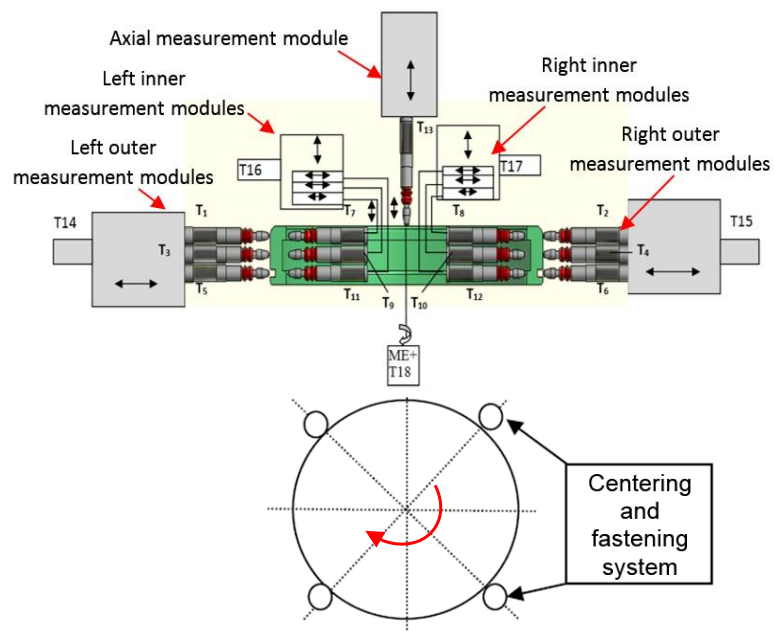

Figure 6. Probes arrengement and part centering in four points

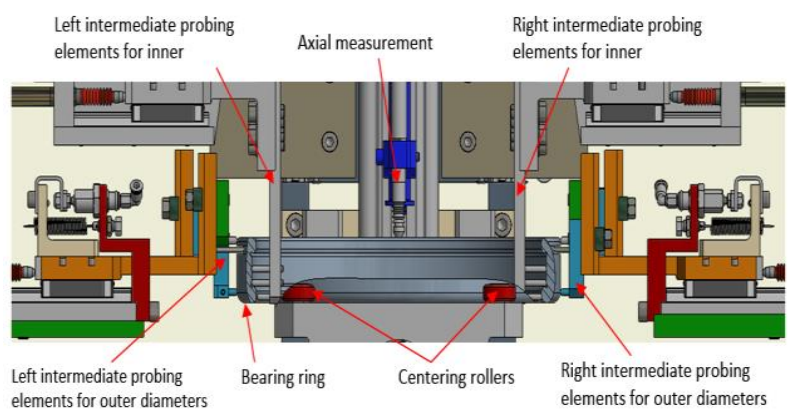

Figure 7. Probing elements

Retraction of the contact probes from part surfaces, at the end of the measurement cycle, is done with miniature pneumatic cylinders with a stroke of $10 \mathrm{~mm}$. The purpose is to protect the probes when the ring is placed into the measuring station or when it is removed. The upward or downward movement of sub-assemblies for inner diameters measurement and axial measurement are also done pneumatically. The pneumatic scheme contains elements for adjusting the speed of moving parts, position sensors and air silencers.

In order to measure a large range of bearing rings without using a large number of calibration standards as well as for the measurement of the absolute diameters, in parallel with the direction of inner and outer diameters measurement were placed four digital absolute scales $\left(\mathrm{T}_{14} \ldots \mathrm{T}_{17}\right)$ with a measuring range of $100 \mathrm{~mm}$ and a resolution of $0,001 \mathrm{~mm}$. (see figure 6).

\section{Calibration and Experimental Results}

The presented equipment may be used to measurement the outside and inside diameters and circular profiles in maximum three radial sections simultaneous. Also, it can be used to determine the radial width deviation of the bearing rings. The main concept of the measurement method is to place the bearing element on a rotary table, centered on inner 
or outer diameter, rotate the element and capture the data from points on the outer or inner surfaces and on the frontal face, at a specified sample rate.

Inner and outer diameters measurement is based on two points measurement method (figure 8.a). Diameters are measured by the distance between a pair of two probes aligned in opposition to each other and working in tandem at a specified sample rate, while the part is rotated through $360^{\circ}$. An average (mean) value is calculated from the sum of the measured diameters at each angular position.

Axial measurements are made with a single probe which determine the deviation at each angular position (figure 8.b). The variation of inner ring width is determined by difference from maximum and minimum measured deviations.

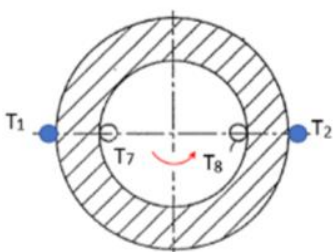

$\mathrm{T}_{1}, \mathrm{~T}_{2}$ - Probes for outside diameter measurement

$\mathrm{T}_{7}, \mathrm{~T}_{8}-$ Probes for inner diameter measurement

a.

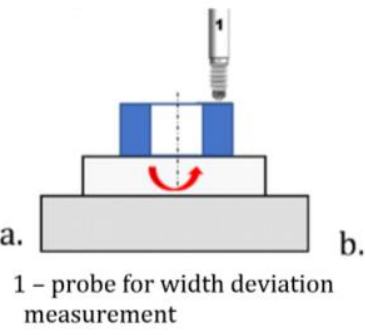

Figure 1. Measurement method for: a. inner/ outer diameters measurement; $b$. radial width deviation measurement

Radial and axial measurements are made with 13 digital linear sensors $\left(\mathrm{T}_{1} \ldots \mathrm{T}_{13}\right)$ with measurement range of $10 \mathrm{~mm}$ (see table 1 and figure 6). These transducers measure only deviations from the nominal value. All intermediate probing elements can be adjusted for measurement of circular profiles into different planes, depending on the form and sizes of the measured part.

Calibration of the equipment is done with a standard ring. The absolute values of the measured outside diameters are calculated based on the value of the outer diameter of the standard, the deviations measured by the transducers pairs $\mathrm{T}_{1}-\mathrm{T}_{2}, \mathrm{~T}_{3}-\mathrm{T}_{4}$ or $\mathrm{T}_{5}-$ $\mathrm{T}_{6}$ and the value measured by the digital linear transducers $\mathrm{T}_{14}, \mathrm{~T}_{15}$.

The absolute values of the measured inner diameters are calculated based on the value of the standard inner diameter, the deviations measured by the pairs of transducers $\mathrm{T}_{7}-\mathrm{T}_{8}, \mathrm{~T}_{9}-\mathrm{T}_{10}$ or $\mathrm{T}_{11}-\mathrm{T}_{12}$ and the value measured by the linear digital transducers $\mathrm{T}_{16}$ and $\mathrm{T}_{17 .}$

So, for the measurement section 1 :

$$
D_{1 i}=C_{1}+T_{14}+T_{15}+\Delta_{T 1}\left(\theta_{i}\right)+\Delta_{T 2}\left(\theta_{i}\right)
$$

$$
\begin{aligned}
& D_{1}=\frac{\sum_{i=1}^{n} D_{i 1}}{n}=C_{1}+T_{14}+T_{15}+\frac{\sum_{i=1}^{n}\left(\Delta_{T 1}\left(\theta_{i}\right)+\Delta_{T 2}\left(\theta_{i}\right)\right)}{n} \\
& d_{1 i}=C_{2}+T_{16}+T_{17}+\Delta_{T 7}\left(\theta_{i}\right)+\Delta_{T 8}\left(\theta_{i}\right) \\
& d_{1}=\frac{\sum_{i=1}^{n} d_{i 1}}{n}=C_{2}+T_{16}+T_{17}+\frac{\sum_{i=1}^{n}\left(\Delta_{T 7}\left(\theta_{i}\right)+\Delta_{T 8}\left(\theta_{i}\right)\right)}{n}
\end{aligned}
$$

where:

$\mathrm{D}_{1 \mathrm{i}}=$ outer diameter in section 1 , measured at angle $\theta_{\mathrm{i}}$ $\mathrm{i}$ = number of sample points for a complete rotation $\left(360^{\circ}\right)$

$\mathrm{C}_{1,2}=$ constants that take into account the position of the scales $\mathrm{T}_{14}, \mathrm{~T}_{15}$ respectively $\mathrm{T}_{16}, \mathrm{~T}_{17}$ relative to the plane containing the rotation axis and perpendicular to the measuring direction; they are determined experimentally.

Similarly are determined the diameters in sections 2 and 3.

Calibration scheme for outer diameters measurement is presented in figure 9.

Initially, the equipment calibration was performed using a standard ring with inner diameter of $45 \mathrm{~mm}$ and outside diameter of $85 \mathrm{~mm}$. This standard ring was measured in metrology laboratory with CMM measurement machine (Leitz Reference 600) and roundness measurement machine (Roncorder EC 2500). (see table 2 and figure 13)

To determine the coefficients $\mathrm{C}_{1}$ and $\mathrm{C}_{2}$, were used the pairs of probes $\mathrm{T}_{3}-\mathrm{T}_{4}$ and $\mathrm{T}_{9}-\mathrm{T}_{10}$.

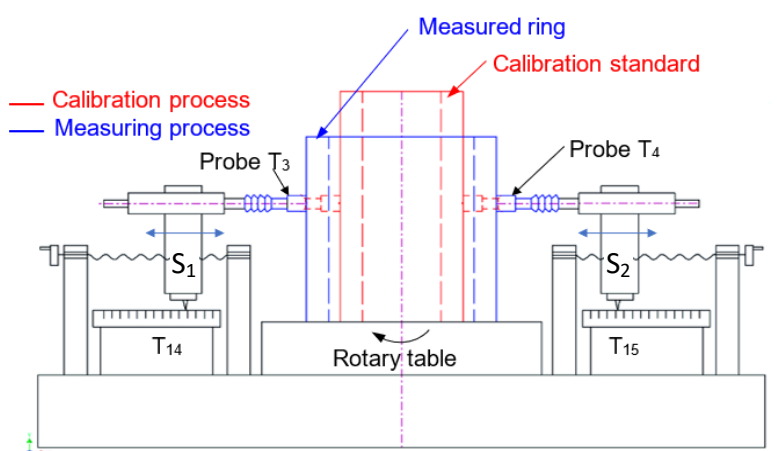

Figure 2. Scheme of calibration and measuring processes

The equipment allows operation in 2 working modes: manual and automatic. The manual working mode allows adjustment and calibration of the equipment, ensuring the following functions (figure 10): actuating the pneumatic cylinders for retracting and returning the radial probes $\mathrm{T} 1 . . . \mathrm{T} 12$ on the part; pneumatic lowering and lifting of the vertical carriage witch are supporting the inner and axial 
measurement modules; rotary table driving; reset of T1...T13 transducers.

For calibrating the outer and inner diameters measurement modules, standard ring was placed on rotary table and centered on inner diameter. The horizontal sledges $S_{1}$ and $S_{2}$ (figure 9) were moved until the probes for outer diameters measurement touched the standard ring. The transducers T14 and T15 must indicate approximately the same value. This is the value at which the calibration of the equipment will be done whenever required, with the mentioned standard ring. Then, the sledges $\mathrm{S}$ have been locked. The transducers T1...T6 have been set up in the measurement field, and then they were locked.

Similarly, were adjusted transducers T7...T2 for measuring inner diameters, moving the slides which are carrying the readheads of the T16 and T17 scales and modules for inner measurements.
Then the values of the transducers T1...T12 have been reset. By switching to automatic mode, a set of measurements was made. The menu for automatic mode measurement of an inner bearing ring is shown in figure 11.

The values of coefficients $\mathrm{C}_{1}$ and $\mathrm{C}_{2}$ were determined as differences between the values of the measured diameters D1 and $\mathrm{d} 1$ and the values of outers, respectively inner diameters of the standard measured in metrology laboratory with Leitz Reference 600 CMM measuring machine and roundness measurement machine Roncorder EC 2500.

Results: Value of $\mathrm{T}_{14}$ and $\mathrm{T}_{15}$ scales for calibration with standard 1 (table 2) = -26,5 $\pm 0,003 \mathrm{~mm}$

Value of $\mathrm{T}_{14}$ and $\mathrm{T}_{15}$ scales for calibration with standard 1 (table 2) = -36,5 $\pm 0,003 \mathrm{~mm}$

$$
\mathrm{C}_{2}=129,091 ; \mathrm{C}_{1}=148.523
$$

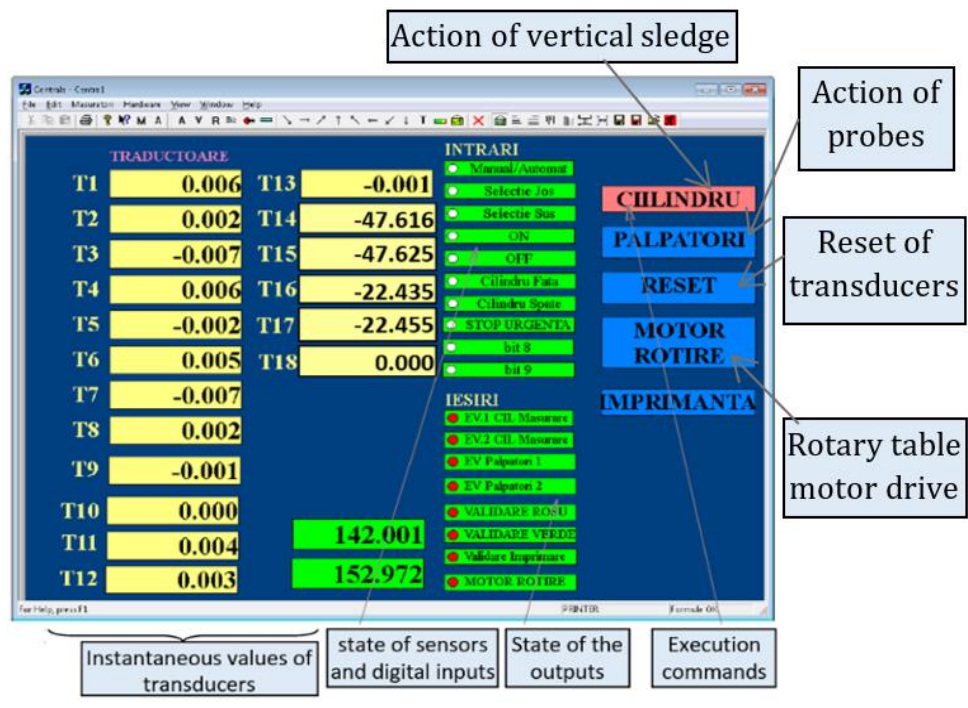

Figure 3. Menu for manual working mode

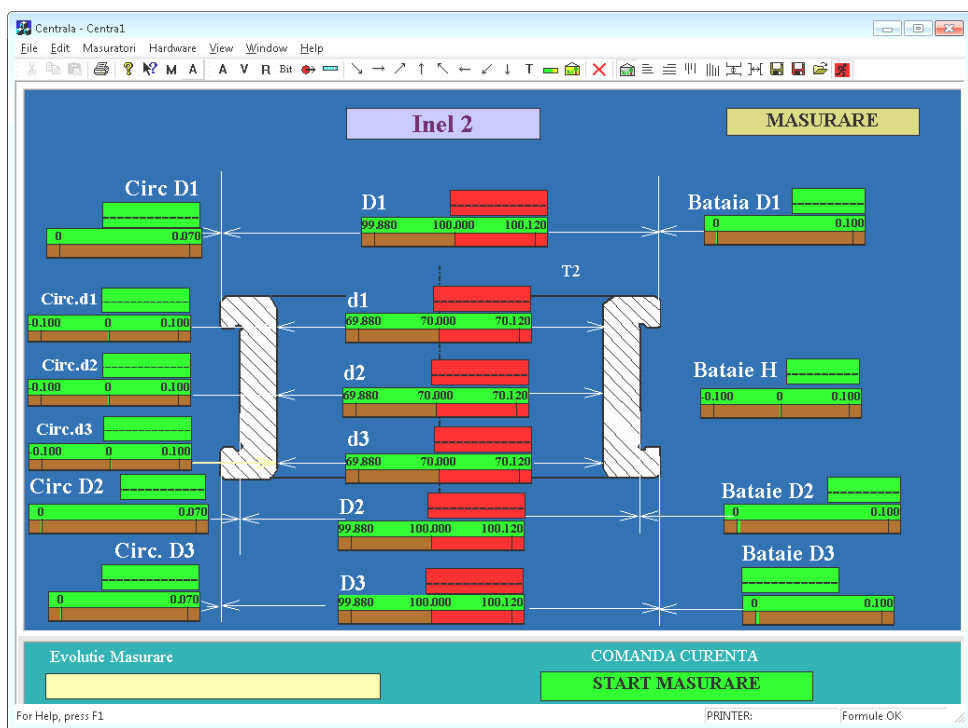

Figure 4. Menu for automatic measurement mode, for an inner bearing ring 
Experimental results have shown that a single standard is not sufficient to achieve the desired accuracy $( \pm 0,01 \mathrm{~mm})$ across the entire measuring range.

Based on the analysis of the measurement error distribution (figure 12) of 11 rings, in two sections $\left(\mathrm{h}_{1}=10 \mathrm{~mm}\right.$ and $\left.\mathrm{h}_{2}=17 \mathrm{~mm}\right)$ the measurement range $(200 \mathrm{~mm})$ was divided into 3 sections and 3 standards were made (one for each section):

Standard 1: $d=45 \mathrm{~mm}$; $\mathrm{D}=85 \mathrm{~mm}$ - for inner measurement range of $45 \ldots 70 \mathrm{~mm}$ and outer measurement range of $50 \ldots 110 \mathrm{~mm}$
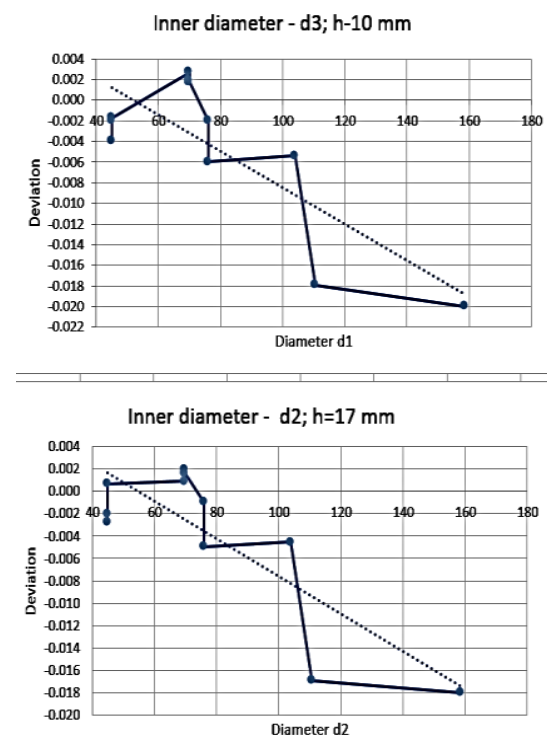

Figure 5. Error distribution charts
Standard 2: $\mathrm{d}=70 \mathrm{~mm}$; $\mathrm{D}=110 \mathrm{~mm}$ - for inner measurement range of $70 \ldots 105 \mathrm{~mm}$ and outer measurement range of $110 \ldots 145 \mathrm{~mm}$

Standard 3: d=105 mm; D=145 mm - for inner measurement range of $105 \ldots 180 \mathrm{~mm}$ and outer measurement range of $145 \ldots 200 \mathrm{~mm}$

For each of these ranges, were determined the values of the $C_{1}$ and $C_{2}$ coefficients and the values of the $\mathrm{T}_{14} \ldots \mathrm{T}_{17}$ scales for calibration.

The dimensions of the standards measured in the metrological laboratory with the Leitz $600 \mathrm{CMM}$ measurement machine and the Roncorder EC 2500 are shown in figure 13 and table 2.

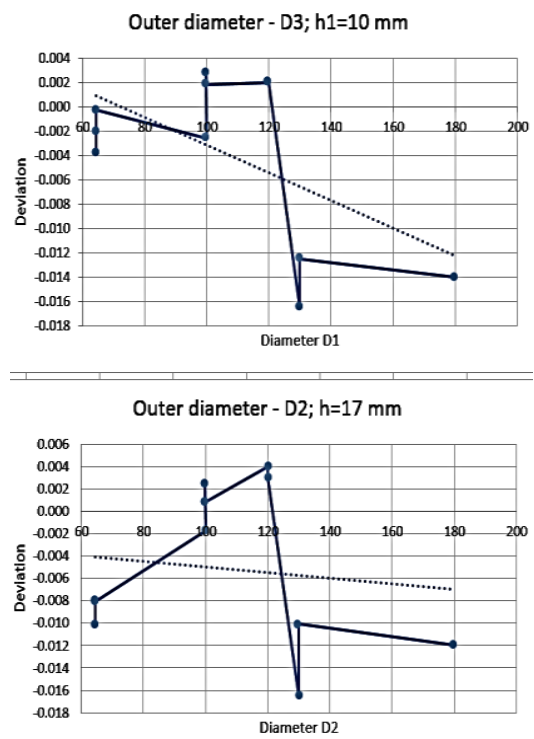

Table 2. Standards dimensions

\begin{tabular}{|c|c|c|c|c|c|c|c|c|}
\hline \multirow{2}{*}{ 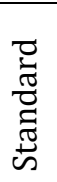 } & \multicolumn{3}{|c|}{$\mathrm{d}(\mathrm{mm})$} & \multicolumn{3}{|c|}{$\mathrm{D}(\mathrm{mm})$} & \multirow{2}{*}{$\begin{array}{c}\begin{array}{c}\text { d Roundness } \\
(\mathrm{mm})\end{array} \\
\mathrm{h}_{2}=17 \\
\mathrm{~mm}\end{array}$} & \multirow{2}{*}{$\begin{array}{c}\text { D Roundness } \\
(\mathrm{mm})\end{array}$} \\
\hline & $\mathrm{h}_{1}=10 \mathrm{~mm}$ & $\begin{array}{c}\mathrm{h}_{2}=17 \\
\mathrm{~mm}\end{array}$ & $\begin{array}{c}\mathrm{h}_{3}=37 \\
\mathrm{~mm}\end{array}$ & $\begin{array}{c}\mathrm{h}_{1}=10 \\
\mathrm{~mm}\end{array}$ & $\begin{array}{c}\mathrm{h}_{2}=17 \\
\mathrm{~mm}\end{array}$ & $\begin{array}{c}\mathrm{h}_{3}=37 \\
\mathrm{~mm}\end{array}$ & & \\
\hline 1 & 45,0032 & 45,0031 & 45,0015 & 85,003 & 85,0026 & 85,003 & 0,0007 & 0,0033 \\
\hline 2 & 69,9954 & 69,9957 & 69,9953 & 110,008 & 110,007 & 110,009 & 0,0015 & 0.0016 \\
\hline 3 & 104,9910 & 104,991 & 104,992 & 145,002 & 155.006 & 145.009 & 0.0018 & 0.0032 \\
\hline
\end{tabular}

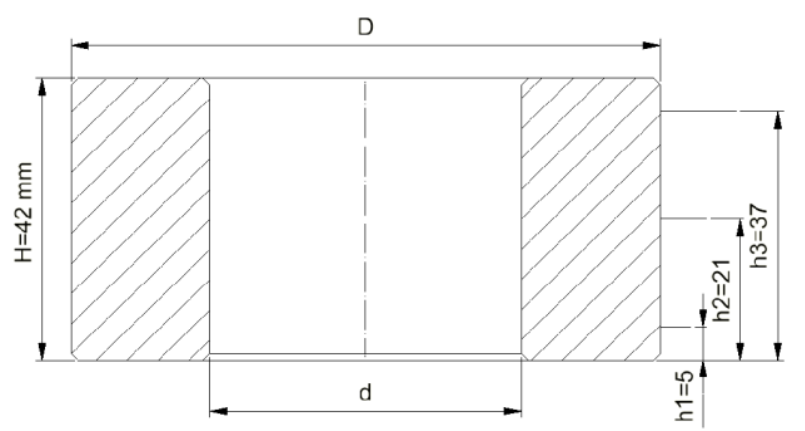

Figure 6. Standard rings
As we mentioned, this equipment may be used also to measurement the roundness of inner and outer bearing rings circular profiles.

The least square method is the most commonly used in practice for roundness measurement. Measurement software accuracy is influenced by various factors: number of measured points, workpiece centering precision, the tilt of cylindrical part's axis relative to the axis of rotation [4] (see figure 14), the filters used, workpieces dimensions. [7]. 
The measured radial data include both the radial form error of the part and the radial errors of the rotary table spindle. Eccentricity and tilt of cylindrical part's axis relative to the axis of rotation of the table influences the accuracy of the diameters measurement (see figure 14). [4]

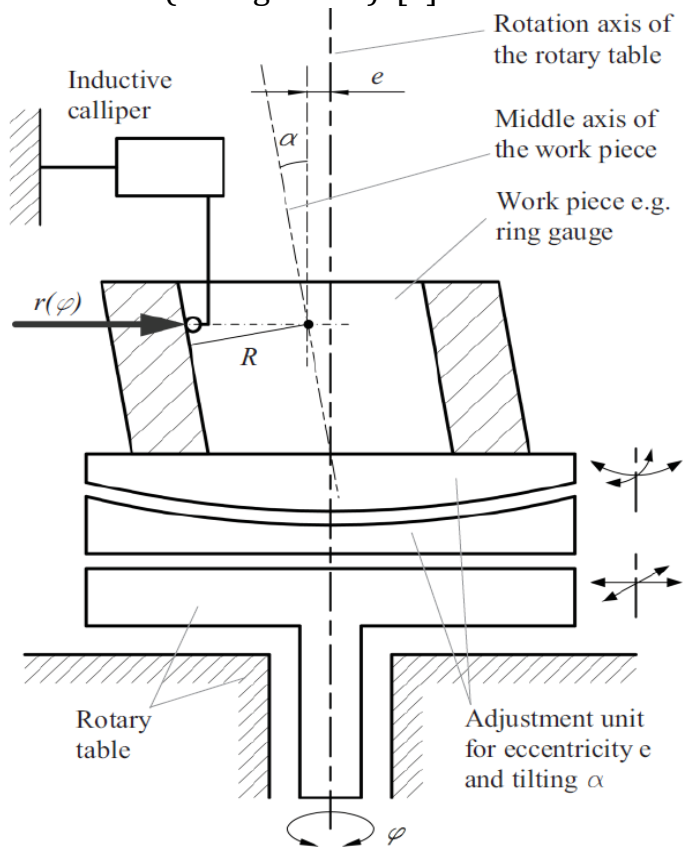

Figure 14: Influence of eccentricity and tilt of cylindrical part's axis on the measurement results

Error separation is important for reducing the measurement errors.

The rotary table spindle has random and systematic components to its radial error motion. We use the reversal method to isolate and remove the systematic component of the radial errors of the rotary table movement. This method for separating part error from spindle error involves measuring the part in two positions of the probe and the part (Bryan et al., 1967; Donaldson, 1972) [5]

For rotary table error separation we used the pair of probes T3, T4 (figure 6) and the standard 1 (Table 2). First the standard ring was centered on inner diameter on the rotary table in a predetermined position, and a set of measurement data was acquired while the table was rotated through $360^{\circ}$. We considered only measured data of $\mathrm{T} 4$. Then, the standard ring was rotated by $180^{\circ}$ and a new set of data was acquired, with the same starting position of the rotary table as in step 1 . We considered only measured data of T3.

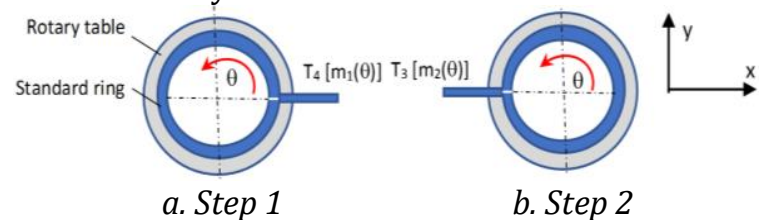

Figure 15. The reversal method for separating part error from spindle error
If $\operatorname{Sx}(\theta)$ is the rotary table error along the probing direction and $R(\theta)$ is the standard ring error, then, in step 1 (Figure 15.a), the probe T4 reads a measured signal $m 1(\theta)$ given by:

$$
m_{1}(\theta)=R(\theta)+S_{x}(\theta)
$$

In the step 2 (Figure 15.b), the probe T3 reads a measured signal $\mathrm{m} 2(\theta)$ given by:

$$
\mathrm{m}_{2}(\theta)=\mathrm{R}(\theta)-\mathrm{S}_{\mathrm{x}}(\theta)
$$

The standard ring and rotary table errors can be obtained from equations (5) and (6):

$$
\begin{aligned}
& R(\theta)=\left(m_{1}(\theta)+m_{2}(\theta)\right) / 2 \\
& S_{x}(\theta)=\left(m_{1}(\theta)-m_{2}(\theta)\right) / 2
\end{aligned}
$$

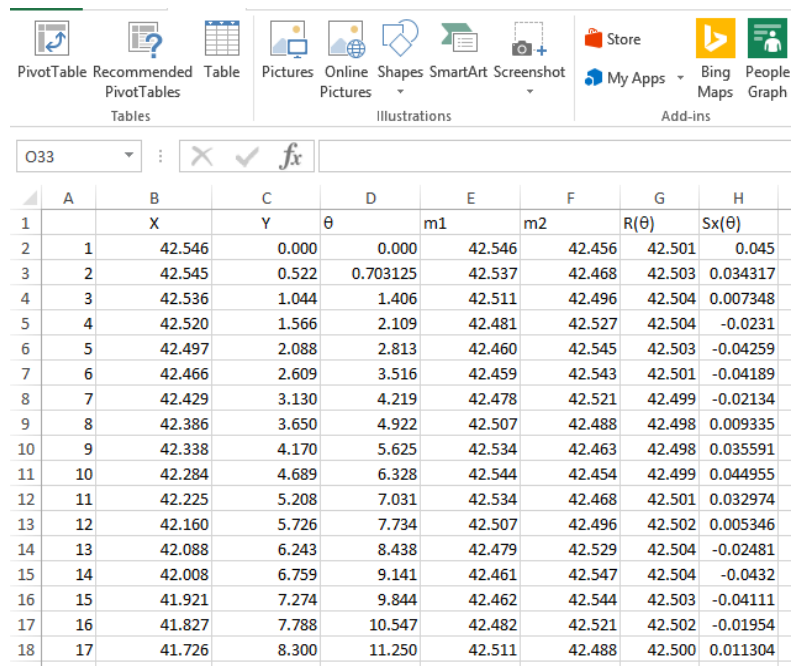

Figure 7. Radial errors of the rotary table spindle

\section{Conclusions}

In this paper was presented a multifunctional equipment for measurement rotary symmetrical parts like bearing rings. The measurement of multiple diameters on a rings with two or more inner and outer cylindrical features, incorporating devices with multiple probes, offer convenience, speed, and economy. By integrtating multiple gaging stations into a single equipment, it is possible to eliminate the expense of duplicate work-holding devices, reducing inspection time and data processing. The operator need fixture the part once and can quickly measure all features in the same time.

The presented equipment meet the following requirements: rapid and precise calibration; a small number of standards for calibration; rapid adaptation to the requirements of the manufacturing process; individual adjustment of the probes in radial and axial direction; multi-parametric control of a wide range of bearing rings; simultaneous measurement of multiple parameters in multiple horizontal planes; decisional capacity based on predetermined criteria; statistical calculation 
program for control and streamlining of manufacturing process.

To increase measurement accuracy the measuring range was divided into three areas, for each of them being determined the calibration parameters. To increase the accuracy of the roundness measurement was performed separation of part errors from rotary table errors.

\section{Acknowledgement}

This work was co-financed by the European Regional Development Fund, through the Competitiveness Operational Programm under the project POC-A1-A1.1.4-E-2015 "Knowledge Transfer Partnerships to Enhance Business Competitiveness in the Field "Automotive Industry and Components" and Improve Road Traffic Safety - KTAutoComp", subsidiary contract 2791/2018".

\section{References}

[1] Piotr Czajka, Tomasz Samborski, Piotr Garbacz, Jordan Mężyk, Automatisation of multiparametric quality inspection of rotary symmetrical metal elements, Journal of Machine Construction and Maintenance, No.2/2017, pp.35-43;

[2] Cristea Luciana, "The Improvement of Performances in Automatic Dimensional Inspection for Bearing Production, an Important Way to Quality Assurance in Mechanical Engineering", Proceedings of the 8th WSEAS International Conference on Instrumentation, Measurement, Circuits and Systems, 2009, 79-82;
[3] Gabriela Bucur, Adrian Moise, Ion Pană, Dragomir Orhei, "Integrating PLCs in Automatic Systems for Dimensional Inspection. CNC Bearings Rings Inspection“, 8th Edition, Electronics, Computers and Artificial Intelligence, 2016, DOI: $10.1109 /$ ECAI.2016.7861186

[4] Michael Kuhnel, Uwe Gerhardt, Vinzenz Ullmann, Eberhard Manske, Automated setup for nontactile high-precision measurements of roundness and cylindricity using two laser interferometers, Measurement Science and Technology 2012, DOI: 10.1088/09570233/23/7/074016;

[5] Bala Muralikrishnan, Jay Raja, “Computational Surface and Roundness Metrology",Springer, 2009, DOI:10.1007/978-1-84800-297-5, ISBN 9781848002968;

[6] Daniela Cioboata, Octavian Dontu2, Daniel Besnea , Robert Ciobanu , Adrian Soare, „Mecatronic Equipment for Bearing Ring Surface Inspection", The Romanian Review Precision Mechanics, Optics \& Mechatronics, 2015, Issue 48, pp 262-266;

[7] Daniela Cioboată, Doru Dumitru Palade, Dănuţ Stanciu, Aurel Abălaru, "Considerations Regarding Roundness Measurement of Closed Profiles and Open Profiles", U.P.B. Sci. Bull., Series D, Vol. 75, ISS. 2, 2013, pp 129-140, ISSN 14542358. 